\title{
Dietary exposure to chemicals within the process of risk assessment: possible applications to substances that may cause allergic reactions
}

\author{
D. Arcella, C. Le Donne and C. Leclercq* \\ National Research Institute for Food and Nutrition, Via Ardeatina 546, 00178-Rome, Italy
}

\begin{abstract}
Exposure assessment is one of the key parts of the risk assessment process. This task is crucial when evaluating substances for which only intake of toxicologically-important amounts can lead to adverse health effects. Ideally, dietary exposure to hazardous substances can be assessed by combining data on concentration in all food products with data on their consumption. However, it is considered to be neither cost-effective nor necessary to collect detailed data for every substance, and a stepwise procedure is commonly used to focus resources on the most important issues. Screening methods, designed to look for 'worst case' situations, are first used to target chemicals that might be of health concern for the general population or for certain at-risk groups. The quality of the dietary exposure assessments not only depends on the quality of the data collected, but also on the integration tools used for initial screening or for the eventual more precise estimations. A particular challenge is the evaluation of food allergens and components causing other forms of intolerances, since no reliable data seem to be currently available on the type of exposure (amounts and duration) required to induce a food allergy. A different approach from that used for dietary exposure to other hazardous substances has to be adopted. However, the methodologies (such as those used to collect food consumption data) and databases (in particular, information about food labels) developed in such a context could be useful to investigate the exposure conditions leading to the development of food allergies.
\end{abstract}

Exposure assessment: Intake: Risk assessment: Allergy

Food does naturally contain, intentionally or unintentionally, a wide range of substances that may constitute a health hazard. In this context risk assessment provides the scientific foundation for the risk analysis process. Risk assessments involve a four-step process: hazard identification; hazard characterisation; exposure assessment; risk characterisation (Codex Alimentarius Commission, 2004). The first stage of a risk assessment, hazard identification, is primarily a question of identifying the effects that are considered as adverse, irrespective of the dose needed or the specific mechanism involved to elicit this effect. The next step, hazard characterisation, is centred on the quantification of these effects, whereas dietary exposure assessment is the qualitative and/or quantitative evaluation of the likely intake of biological, chemical or physical agents via food. Risk characterisation is the final stage of risk assessment that integrates information from exposure assessment and hazard characterisation into advice suitable for use in decision-making.
A particular challenge is the evaluation of food allergens and components causing other forms of intolerances, as very little data seem to be currently available on the type of exposure (amounts and duration) required to induce a food allergy. Clearly, a different approach from that used for dietary exposure to hazardous substances has to be adopted. However, the methodologies (such as those used to collect food consumption data) and databases (particularly information about food labels) developed in such a context could be useful in investigating the exposure conditions leading to the development of food allergies.

The aim of the present paper is to illustrate the process currently used to estimate the exposure to hazardous chemicals from the diet. In particular, statistical methods and databases commonly used for dietary exposure assessment will be described, highlighting those that could be useful within the process of risk assessment of substances that may cause allergic reactions.

*Corresponding author: Dr Catherine Leclercq, fax + 3906 51494550, email leclercq@inran.it 


\section{Current databases and methods used in dietary exposure assessment}

Dietary exposure assessment is a crucial component of risk assessment applied to chemical substances in foods and beverages. In order to calculate reliable estimates of the amounts ingested through the diet for a specific chemical substance, three elements have to be taken into account: (1) levels and fate of the chemical in food; (2) food consumption patterns; (3) integration of these elements to determine exposure. In all three areas the limitations of the approaches currently used lead to uncertainties that can either cause over- or underestimation of real intakes and thus of risk. The basic components for dietary exposure assessment will be discussed.

\section{Presence and levels of chemicals in food}

Factors influencing the variability of chemical concentration levels in ready-to-eat food products are legislation, agricultural and storage practices, and processing. Quantitative information on the level of a chemical in food can be obtained through analytical determination. However, errors, wrong assumptions or inaccurate measurements can lead to results differing markedly from the real concentration of the chemical in food when the amount is determined analytically. In particular, food sampling procedures for subsequent analysis can critically determine how close the measured value is to the real value. Aspects such as climate, ripeness, soil conditions etc. are likely to influence whether a chemical is present and should be considered when food products are sampled for analysis and methods are chosen. For example, the presence of pesticide residues in crops and derived foods is related to climatic differences and yearto-year variation (van Klaveren, 1999). Once representative samples are taken, the accuracy of the analytical methods used is important. In particular, analytical methods used for screening purposes or for the evaluation of substances present at high levels tend to be less precise than methods used for accurate quantification, especially at low levels of detection. Moreover, chemical levels can be measured at a much later stage in the food production chain than when the chemical is first introduced. Thus, a further source of uncertainty is linked to the possible effects of food processing (e.g. washing, peeling or cooking) on the chemical of interest, as a result of industrial and/or household preparation (Kroes et al. 2002).

When evaluating the presence and levels of chemicals in food, it is valuable to distinguish between substances intentionally added (particularly additives and flavourings) and other substances (e.g. pesticides residues and contaminants). In the case of intentionally-added substances, the information about the presence is generally indicated on the product label and occurrence data can be obtained from the legislation regulating the use of these substances.

Additive usage levels are regulated at the European level (European Commission, 1994a,b,c, 1995). The general criteria for the use of food additives is that they perform a useful purpose, are safe and do not mislead the consumer.
In highly-processed foods, particularly, additives are used to standardise product parameters and stability in order to achieve the required shelf-life up to and after purchase by the consumer, while maintaining the product properties expected by the consumer. While some products with a long shelf-life are dependent on the use of additives, it should be recognised that some additives also decompose with time so that the levels present at the end of shelf-life may be comparatively low. An example is antioxidants, which are designed to stabilise products by interacting with $\mathrm{O}_{2}$ that is present in the product or permeates through the packaging. In such cases it is important to understand which decomposition products are likely to be formed so that they can be considered in exposure and risk assessments. Most foods contain only a small number of all additives permitted for a given category, particularly if alternative substances with the same function, or alternative approaches, are available. For example, suitable process or storage conditions may help to avoid or reduce additive use. As a consequence, additive levels may range from the maximum permitted level to none. For example, the sulphite content of wine is generally lower than the corresponding maximum permitted level $(200 \mathrm{mg} / \mathrm{l})$; furthermore, its level has decreased in the last decades probably because new technological processes have led to improved wine stability at a lower sulphite level. In the 1980s a large survey of wines was conducted in Europe, in which 3655 samples of Italian wine were analysed. Their mean $\mathrm{SO}_{2}$ content was found to be $135 \mathrm{mg} / \mathrm{l}$ (Ough, 1986), compared with $92 \mathrm{mg} / \mathrm{l}$ reported in a more recent study (Leclercq et al. 2000b). Levels of sulphite in wine have been found to be even lower in France (75 mg/l; Mareschi et al. 1992).

Moreover, when, as is normally the case, several additives with the same function are permitted, they can be used either as alternatives or as simple mixtures. In some cases, e.g. the intense sweeteners aspartame and acesulfame $\mathrm{K}$, additives may show synergistic action that not only provides an improved end result (sweetness profile in the case of the sweeteners) but permits the reduction of the amounts needed for each individual component.

Flavouring substances do not follow the same form of regulation as food additives. In the EU, in which industry currently claims to be adding $>2800$ different chemically-defined flavouring substances (European Commission, 1996, 2002), their safety is currently being evaluated. A register of these flavourings has been established, and the substances listed are to be assessed for their safety in use according to the evaluation programme laid down in Commission Regulation (EC) 1565/2000 (European Commission, 2000b). After the completion of the evaluation programme a definitive list of flavouring substances for use in or on foods in the EU will be adopted (Article 5 (1) of Regulation (EC) 2232/96; European Commission, 1996).

Individual flavour components are usually not specifically indicated on the label beyond the statement that flavours are used, and maximum levels have not been established, with the exception of some specific flavouring substances such as cyanogenic substances (European 
Commission, 1988). European law only requires the use of the word 'flavouring' or the specific name or description of the substance (e.g. vanilla) on the label. The word 'natural' may be added only for flavourings for which the component contains exclusively flavouring preparations that have been isolated by appropriate physical processes (enzymic or microbiological) or traditional foodpreparation processes (European Commission, 1988).

Another category of substances that are intentionally added during food processing is enzymes. According to the European Directive 95/2/EC (European Commission, 1995) enzymes can also be classified as food additives (e.g. invertase E1103 and lysozyme E1105) and labelled in the same way as any other food ingredient (European Commission, 2000a). However, enzymes are predominantly used as processing aids, as they have a technological role only during processing and they should not be present in the final product. In the EU there is still not a directive to regulate the use of enzymes as processing aids in foodstuffs, although there is regulation for the use of enzymes only for fruit juices (European Commission, 1993) and wine (European Commission, 1999). Some member states currently have national legislation to regulate the use of these substances.

Data on patterns of use of chemical substances intentionally added to food are difficult to obtain. Information on concentrations in brand foods is considered to be commercially sensitive and is therefore not widely available. Additives, like all other ingredients, must be included on the product label in descending order of level. This information may indicate the presence of the additive and in some cases the amounts used, but collecting such data from labels is time-consuming.

In order to provide all consumers with better information and to protect the health of particular groups of consumers, the European Commission has amended the legislation relating to the labelling of foodstuffs (European Commission, 2000a). Thus, the European Parliament (European Commission, 2003) has abolished the ' $25 \%$ rule', under which individual ingredients making up a compound ingredient did not have to be listed if the compound ingredient made up $<25 \%$ of the final food product. According to the new directive all ingredients intentionally added have to be included on the label, and the new threshold for such exemption is now $2 \%$. The directive also contains a list of twelve ingredients liable to cause allergies or intolerances. Any ingredient listed in the directive or originating from an ingredient listed in the directive that is used in production of a foodstuff and is still present in the finished product, even if it is in an altered form, must be indicated on the label with a clear reference to the name of this ingredient. The list of ingredients liable to cause allergies or intolerances comprises only one chemical substance, $\mathrm{SO}_{2}$ and sulphites at concentrations of $>10 \mathrm{mg} / \mathrm{kg}$ or $10 \mathrm{mg} / \mathrm{l}$ expressed as $\mathrm{SO}_{2}$. In the case of alcoholic beverages it will now be mandatory to include in the labelling all ingredients present in the beverage that may have an allergenic effect.

The European Food Safety Authority (2004) has considered that certain products or ingredients are not likely, or not very likely, to cause adverse reactions in susceptible individuals. These products or ingredients are therefore included in a list of products provisionally exempt from allergen labelling until November 2007 (European Commission, 2005).

Food label databases have been developed in order to take advantage of the information present on the food labels within the task of exposure assessment to hazardous chemicals. In The Netherlands, for example, a foodhypersensitivity databank (the ALBA databank; TNO Nutrition and Food Research, 2004) has been developed to store information on the composition of foods in terms of the presence or absence of certain components acknowledged as being responsible for food intolerance or food allergy. The food companies provide data on a voluntary basis and this information is available to consumers. In 2004 the ALBA databank was used for the identification of relevant foods and beverages containing wheat-starch hydrolysates in a study aimed at assessing dietary exposure to gluten through the dietary use of the wheat-starch hydrolysates (glucose syrup, dextrose and maltodextrin; RA Bausch-Goldbohm, HF Balder, D Arcella and S McCarthy, unpublished results). Another example of a food label database for processed foods is that created by the Italian National Research Institute for Food and Nutrition (2003). This database is not being updated on a systematic basis, but information relating to categories of foods is included in the database according to the needs of specific projects aimed at assessing dietary intake of food additives. This database has been used to estimate the potential dietary intake of antioxidant additives (erythorbic acid, gallates, butylated hydroxyaniline and butylated hydroxytoluene) in Italy (Leclercq et al. 2000a). More recently, the Italian food label database was used in a study that aimed to assess the intake of intense sweeteners in a sample of teenagers living in the district of Rome (Arcella et al. 2004), in which all the labels of packaged products consumed during the survey and thought to contain intense sweeteners were included. Furthermore, the average concentration of the intense sweeteners for each sugar-free product, provided by producers, was recorded in the database. In the USA food label information has been collected within the Food Label and Package Survey conducted by the Center for Food Safety and Applied Nutrition of the Food and Drug Administration. Data from the 2000-2001 Food Label and Package Survey characterise various aspects of the labelling of processed packaged foods, including nutrition labelling and various types of label claim (Legault et al. 2004).

Substances not intentionally added that may be present in foods and beverages, depending on specific conditions, include pesticides, naturally-derived components (such as mycotoxins or geologically-derived levels of heavy metals) and man-made chemicals from different sources, including environmental pollutants and contaminants derived from animal feed or plant fertilisers. In these cases the presence and levels in foods are primarily derived from analysis of raw agricultural commodities during routine monitoring, enforcement monitoring or import monitoring programmes. Thus, many monitoring programmes relating to toxins and contaminants focus on those samples that 
may contain residue levels above the permitted limit (e.g. those products produced during wet seasons, under difficult conditions or out of season) and are therefore often not random. Another category of residues is substances that migrate from packaging materials into the food.

For pesticides and for most environmental contaminants an important source of uncertainty is the fact that sampling procedures in fruit and vegetables usually include the preparation of composite samples (i.e. ten apples, $2 \mathrm{~kg}$ lettuce) to be homogenised for analysis (Kroes et al. 2002). Results are therefore always presented as the mean of the residues present in the composite sample. However, for some food commodities the consumer eats only a small number of units daily compared with those of a composite sample and all chemicals found may originate from only one or two units. For example, the concentration in a single carrot may have been ten times higher than the concentration found in a composite sample comprising ten carrots. This factor could be crucial when the exposure to a substance is compared with its acute reference dose.

Another important limitation is that analyses of residues in fruit and vegetables are mainly conducted using raw agricultural commodities, which include the skin and nonedible parts. Processed or prepared foods are either not monitored or the number of samples is very small, because in the legislation the limits for residues are mainly set for raw agricultural commodities and the purpose of monitoring is usually to assess compliance rather than exposure.

\section{Food consumption patterns}

When assessing exposure four different sources of information on food consumption can be used: food supply data; data from household consumption surveys; data from dietary surveys for individuals; the collection of duplicate diets. Biomarkers form a fifth source of exposure data, whereby these measures reflect both the consumption of food and the concentration of the chemical in these foods.

Disappearance data provide gross annual estimates of the national availability of food commodities. Food supply data are calculated in food balance sheets, which are accounts, on a national level, of annual production of food, changes in stocks, imports and exports, agricultural use and industrial use (Kroes et al. 2002). The result is an estimate of the average supply per capita of the population, irrespective of, for example, age or gender. Food supply data relate to food availability, which gives only a crude (usually overestimated) indication of potential average consumption. Food losses before consumption, as a result of processing, spoilage, trimming and waste, may not be accounted for adequately. As a result of their long history, food balance sheets are used particularly for assessing trends over time. International food balance sheets are prepared and published by FAO (for example, see Food and Agriculture Organization, 2003), while EUROSTAT (see EUROSTAT, 2005) publishes data for gross human apparent consumption of main food items for the EU member states using similar methodology.

Food available at the household level may be estimated from budget surveys and consumption surveys. The budget survey gives information on the purchases of food in terms of expenditure and is used for economic policy. In household consumption surveys the amounts of foods and drinks brought into the household are also recorded, but not usually for meals consumed out of home. In general, household surveys do not provide information on how food is handled within the household, or on actual consumption by its members. Data on the quantity of and/or expenditure on food may be collected by record keeping and/or by interview. The Data Food Networking (Trichopoulou, 2001) project is harmonising the data from household budget surveys, focusing on the creation of a pan-European food databank based on national household budget surveys by the development of the most appropriate way of using food and related data from these surveys.

The data from dietary surveys among individuals more closely reflect actual consumption. Several methods can be used for the collection of dietary intake data at an individual level, which can be divided into two categories, record and recall. Record methods collect information on current intake over $\geq 1 \mathrm{~d}$. Recall methods reflect past consumption, varying from intake over the previous day $(24 \mathrm{~h}$ recall) to usual food intake (dietary history or food frequency). Several European countries have performed individual surveys on a national basis (EFCOSUM Group, 2001). The duplicate-diet method differs from the other methods in that the intake estimation does not rely on composition data from other data sources. The concentration is measured by chemical analysis of the duplicate diet collected.

The quality of the results of a food-chemical exposure assessment will depend largely on the quality of the food consumption data used in the assessment. Collecting food consumption data in surveys as well as in duplicate diet samples for exposure assessment can alter the diet of study participants, resulting in a bias in intake estimate. Intake may also be altered if participants change their diet as a result of fatigue or loss of interest (Kroes et al. 2002). It is important to highlight that many dietary surveys have reported energy intakes that are consistently lower than the levels that would be expected from the estimated basal metabolic expenditure and physical activity. This problem of under-reporting of energy intake is widespread and is one of the major biases in exposure assessments based on dietary surveys (Gilsenan \& Gibney, 2004).

Another important issue is the food coding system. In dietary assessment, food products have to be identified and thereafter categorised in line with the purposes of the study; this has led to the existence of national and international systems, but unconventional categorisation systems are often needed for exposure assessment of a broad range of chemicals.

\section{Methods used in dietary exposure assessment}

Until 10 years ago assessment of dietary exposure was mainly based on the estimates obtained by combining the maximum permitted level with mean food consumption data for all chemicals (Saba et al. 1992). However, mean consumption is not informative in relation to subjects 
consuming high levels of one or more food categories (high-level consumers), who are more likely to ingest hazardous chemicals in excessive quantities. The methodologies now adopted for the assessment of exposure from diet take into special consideration non-average individuals, and in particular those who consume relatively large quantities of foods containing higher concentrations of substances that may potentially lead to a health risk. However, the definition of high-level consumers can vary, it is normally the 90 th, 95th, $97 \cdot 5$ th or 99 th percentile of the distribution of individual intake values. A high percentile, rather than the maximum value, is usually chosen because maximum intakes are unlikely to be maintained over long periods of time and are not therefore representative of high-level intakes in relation to chronic exposure. These considerations imply that the choice of the percentile value and the precision level required have a large impact on the sample size, since the acceptability of the use of percentile values (in relation to the reliability of estimates) depends to a great extent on the sample size.

It is important to emphasise the issue of the definition of high-level consumers because, in practice, it defines the proportion of the population that would have to exceed the at-risk dose before action is considered necessary to reduce exposure. This problem is not only scientific but political and ethical. From a scientific point of view there are several possible approaches to the evaluation of the intake of a substance contained in food, but in many cases there are statistical difficulties in measuring adequate percentiles of intake. Thus, if methodological limitations do not allow the assessment of the 99th percentile of intake, the fact that the 95th percentile of intake is below the safety limit can appear to be reliable, but this result may not always be considered satisfactory. In fact, $1 \%$ of the population at risk of an excessive intake of a certain substance is an important political and ethical problem (European Commission, 1998).

Ideally, dietary exposure to chemical substances should be assessed by combining data on their concentration in all food products with data on their consumption. Unfortunately, with the exception of duplicate-diet studies, exposure assessments are not performed on the basis of consumption, occurrence and concentration data related to the same individuals within a population. Thus, assessments of exposure to dietary components will usually require some extent of modelling in an attempt to create a representation of the real-life exposure situation. There are a number of different models for combining or integrating the consumption data with the residue and/or concentration data, and a number of factors that influence the choice of model for any given exposure assessment, including the purpose of the assessment (target chemical, population group, extent of accuracy required) and the availability of data. In general, it is considered neither cost-effective nor necessary to collect detailed food consumption and chemical concentration data for every hazardous substance (Lawrie \& Rees, 1996). A stepwise procedure is commonly used to minimise estimation costs and focus resources on the most important issues. With the stepwise approach to dietary exposure assessment of food chemicals, as the accuracy of dietary exposure assessments increases, the cost of collecting adequate data and resources needed to undertake the assessments also increases (World Health Organization, 1997). The aim of the stepwise procedure is to target dietary exposure estimates to chemicals that might be of health concern for 'average consumers' or individuals belonging to certain at-risk groups. If the estimated exposure to a given pesticide residue, food additive, veterinary drug residue or contaminant exceeds its safety limit (e.g. acceptable daily intake), a more accurate method of dietary exposure assessment should be applied. Exposure is therefore first assessed using methods that follow a deterministic approach based on conservative assumptions. At this stage, particularly, the methodologies adopted for the assessment of exposure from diet should take into special consideration high-level consumers. However, food consumption data are not commonly available at an individual level for high-level consumers. Consequently, highly-conservative methods are used. It is important to remember that these methods are not suitable for predicting actual exposure because they are designed to include the worst-case scenario. Nevertheless, they are useful and inexpensive screening tools for identifying those substances for which safety limits of intake may be exceeded. The methods currently used include: the 'budget method' (Hansen, 1979), which is mainly used in the field of food additives; the maximised survey-derived intake (Munro et al. 1999) and the theoretical added maximum daily intake (Cadby, 1996) methods, which are used to assess the intake for flavourings; the international estimate of short-term intake (Food and Agriculture Organization/World Health Organization, 2001), which is used for pesticides. In some cases these methods fail in overestimating exposure and should be revised (Arcella \& Leclerq, 2005).

An example of a stepwise procedure that has been adopted to evaluate the safety of intentionally-added chemicals is that performed by Leclercq et al. (2000a) in relation to food additives with primary antioxidant technological function. The potential intake of these additives in Italy has been estimated by means of a hierarchical approach using, step-by-step, more refined methods. The results of the investigations conducted by Leclercq et al. (2000a) suggest that the maximum permitted levels that have been fixed for the EU for erythorbic acid, gallates and butylated hydroxyaniline are adequate to avoid the risk of intake above the acceptable daily intake in Italy, and also for those individuals who would consume relatively large quantities of foods in which these additives are allowed. However, at the end of the three-step procedure it could not be excluded that intakes for butylated hydroxytoluene for a substantial proportion of the Italian population may exceed the acceptable daily intake. This finding does not mean that the legislation for this additive is not intrinsically safe, but that there is a need for a more precise approach that uses analytical data relating to its main potential sources.

When consumption data, but not occurrence data, are available at an individual level, conservative approaches are usually adopted that assume that the chemical is present at the maximum level in all the products in which it is legally permitted. However, consumption data are 
often only available for aggregate food categories that are larger than those defined when establishing the maximum levels, e.g. in most national household budget surveys. Commonly, with this type of data an estimate of the chemical intake is obtained by using the highest chemical concentration value allowed within the category, which is obviously a further conservative assumption.

Recently, in order to achieve a more realistic view of exposure to hazardous substances, risk managers have become more interested in probabilistic modelling (European Commission, 2000c; Petersen, 2000). Probabilistic approaches are typically more resource-intensive than deterministic approaches, but they permit the characterisation of the variability and uncertainty that may exist in such exposure estimates and thus facilitate more meaningful and realistic assessments. The results, however, are only as good as the input data, algorithms and assumptions. Thus, the critical aspects of the food consumption and chemical concentration data used in the assessment of deterministic estimates must also be taken into account when using a probabilistic approach. Moreover, the impact of the assumptions should always be tested carefully and the results should be fully documented. A modelling tool must be structured so that all algorithms and assumptions inherent to the model can be identified and validated. Sensitivity analyses should be used to set priorities for risk-reduction measures and to define the main sources of uncertainty in order to plan further studies and improve the exposure estimates.

\section{Exposure assessment for substances that may cause allergic reactions}

Food allergies must be distinguished from food intolerances. Food allergies are abnormal immunological reactions to a food or food component (Poms et al. 2004). Most other food sensitivities do not involve the immune system and are not considered true food allergies. They may be caused by pharmaceutical reactions to food additives (e.g. benzoates, salicylates, sulphites, or infectious agents such as bacterial or viral products), enzyme deficiencies (e.g. lactose intolerance), diseases of the digestive system (e.g. obstructions), idiosyncratic reactions (individualistic adverse reactions with unexplained mechanisms) to food or even psychological reasons. Another condition involving food sensitivity that is of particular relevance is coeliac disease, also known as coeliac sprue or gluten-sensitive enteropathy (Poms et al. 2004).

Within toxicological evaluations, when estimating the ability of a chemical to cause illness (hazard identification) it is reasonable to assume that any chemical is a potential hazard to man. The susceptibility may vary, but if the dose is high enough all individuals are at risk of health effects. When dealing with hazard identification and risk assessment of substances that may cause allergic reactions it is evident that for the majority of the population there is no hazard, and consequently the risk of allergic reaction is zero even at extreme doses (Madsen, 2001). It has been suggested (Sampson, 1999; Wuthrich, 2000; Ortolani et al.
2001; Sicherer et al. 2003) that in industrialised societies food allergies affect about $1-2 \%$ of the total population and $\leq 8 \%$ of the children, particularly young children.

The individuals affected can only completely avoid their particular problem materials. For the allergic individuals the relevant information relating to exposure is mainly the presence of the allergen in any serving. More quantitative exposure assessments may be unnecessary. In the case of foods known to contain allergens or substances causing intolerances labelling is often sufficient to avoid exposure. Problems can arise when the presence of the relevant allergen is not expected. In fact, total avoidance is sometimes difficult for the allergic individual, since processed food products contain a large variety of ingredients that may include allergenic foods. Sensitive individuals may also be inadvertently exposed to allergenic proteins by consumption of food products that are supposed to be free of a certain allergen. Food products can be contaminated with 'foreign' food constituents during shipping and storage, during processing, from carry-over resulting from inadequate cleaning of shared processing equipment, or through reworking of allergencontaining products (Huggett \& Hischenhuber, 1998).

Few reliable data seem to be currently available on the type of exposure (amounts and duration) required to induce a food allergy, but there are a number of studies that aim to define the threshold of concentration in order to implement analytical protocols. The range of minimum doses of allergen required to trigger a reaction in sensitised individuals has not been defined accurately for any allergen, but is known to vary considerably between individuals (Crevel, 2001). Threshold levels for specific allergic reactions determined by double-blind placebocontrolled food challenges range from $<1 \mathrm{mg}$ to $>1 \mathrm{~g}$ allergenic protein, depending on the food concerned and the sensitivity of selected allergic individuals (Taylor et al. 2002). There is general agreement that the detection limits for different food products need to be between 1 and $100 \mathrm{mg}$ allergenic protein/ $\mathrm{kg}$ food, depending on the food (Poms et al. 2004).

When threshold levels have been determined by human oral-challenge studies it may be appropriate to assess exposure quantitatively. In this case databases and methods applied in dietary exposure assessment could be extremely useful. An example of dietary exposure assessment for substances responsible for allergic reactions that may not be expected to be present on the basis of composition is the study of RA Bausch-Goldbohm, HF Balder, D Arcella and S McCarthy (unpublished results), which was supported by the European Cereals Starch Association. The aim was to assess the exposure to gluten through the dietary use of wheat-starch hydrolysates in foods and beverages that contain glucose syrup, dextrose and/or maltodextrin but do not currently have to be labelled as 'gluten-containing', as would another source of gluten, such as wheat flour. Exposure assessment was performed using food consumption data at the individual level from three European countries: The Netherlands; Italy; the Republic of Ireland and Northern Ireland. Information on the use of (wheat) starch hydrolysates in food and beverages cannot readily be derived from existing databases. Thus, a 
primary selection of relevant food and beverage groups was made, based on sales data provided to the European Cereals Starch Association by the five biggest producers of starch hydrolysates. A secondary selection was based on information on food applications, also provided by European Cereals Starch Association. Independently from these data, information available from the ALBA databank (TNO Nutrition and Food Research, 2004) was used. Crude ranges of the potential content of hydrolysates were derived from the total mono- and disaccharide content of the foods, as available from the Dutch food composition database (NEVO Foundation, 2004) and in some cases complemented by information derived from the label for the food or beverage. For most of the main groups information from producers and/or Dutch or European industry associations was requested so that the range of specific hydrolysate content of the food and beverage subgroups could be refined. For exposure assessment a worst-case scenario and a more realistic scenario were applied, varying the content and source of hydrolysates in foods and beverages and the gluten content of the hydrolysates. This study was not intended to assess adverse effects related to gluten, but only to give an estimate of its potential range of exposure in the general population through the dietary use of wheat-starch hydrolysates in foods and beverages.

The European Food Safety Authority Scientific Panel on Dietetic Products, Nutrition and Allergies was aware of the European Cereals Starch Association research and stated that 'it is unclear how much the study will be able to answer the central question, namely the potential effect of wheat hydrolysates in coeliac disease and in wheat allergy' (European Food Safety Authority, 2004). According to the European Food Safety Authority Scientific Panel on Dietetic Products, Nutrition and Allergies (European Food Safety Authority, 2004) 'wheat-based glucose syrups including dextrose are unlikely to cause an adverse reaction in individuals with coeliac disease provided that the provisional value of gluten considered by Codex Alimentarius for foods rendered gluten-free (currently $200 \mathrm{mg} / \mathrm{kg}$ ) is not exceeded'. Wheat-based glucose syrups including dextrose, wheat-based maltodextrins and products thereof have therefore been included in the list of products provisionally exempted from allergen labelling until 25 November 2007 (European Commission, 2005).

The interaction between the research performed within the field of dietary exposure assessment and the evaluation of food allergens and components causing other forms of intolerances is likely to further increase in the future. In fact, Kroes et al. (2002) have noted that the use of probabilistic modelling could be a promising area for common research challenges. It would be particularly valuable to develop and validate probabilistic models for exposure to allergens and methods in order to predict the probability of adverse effects arising from such exposures.

\section{References}

Arcella D \& Leclercq C (2005) Assessment of dietary intake of flavouring substances within the procedure for their safety evaluation. Advantages and limitations of estimates obtained by means of a per capita method. Food and Chemical Toxicology 43, 105-116.

Arcella D, Le Donne C, Piccinelli R \& Leclercq C (2004) Dietary intake of intense sweeteners by Italian teenagers. Present levels and projections derived from the INRAN-RM-2001 food survey. Food and Chemical Toxicology 42, 677-685.

Cadby P (1996) Estimating intakes of flavouring substances. Food Additives and Contaminants 13, 453-460.

Codex Alimentarius Commission (2004) Joint FAO/WHO Food Standards Programme: Procedural Manual. 13th ed. Rome: FAO/WHO.

Crevel RW (2001) Risk assessment for food allergy - the industry viewpoint. Allergy 56, Suppl. 67, 94-97.

EFCOSUM Group (2001) European Food Consumption Survey Method. TNO Report V3766. Zeist, The Netherlands: TNO Nutrition and Food Research

European Commission (1988) Council Directive 88/388/EEC of 22 June 1988 on the approximation of the laws of the Member States relating to flavourings for use in foodstuffs and to source materials for their production. Official Journal of the European Communities L184, 61-71.

European Commission (1993) Council Directive 93/77/EEC of 21 September 1993 relating to fruit juices and certain similar products. Official Journal of the European Communities L244, $58-66$.

European Commission (1994a) European Parliament and Council Directive 94/34/EC of 30 June 1994 amending Directive 89/ 107/EEC on the approximation of the laws of Member States concerning food additives authorized for use in foodstuffs intended for human consumption. Official Journal of the European Communities L237, 1-2.

European Commission (1994b) European Parliament and Council Directive 94/35/EC of 30 June 1994 on sweeteners for use in foodstuffs. Official Journal of the European Communities L237, 10.9.94, 3-18.

European Commission (1994c) European Parliament and Council Directive 94/36/EC of 30 June 1994 on colours for use in foodstuffs. Official Journal of the European Communities L237, 10.9.94, 13-29.

European Commission (1995) Council Directive No 95/2/EC of 20 February 1995 on food additives other than colours and sweeteners. Official Journal of the European Communities L061, 23.

European Commission (1996) Regulation (EC) 2232/96 of the European Parliament and of the Council of 28 October 1996 laying down a Community procedure for flavouring substances used or intended for use in or on foodstuffs. Official Journal of the European Communities. L299, 1-4.

European Commission (1998) Report on methodologies for the monitoring of food additive intake across the European Union (Final report submitted by the Task Co-ordinator 16 January 1998). Reports of a Working Group on Scientific Co-operation on Ouestions Relating to Food. Task 4.2. SCOOP/INT/ REPORT/2. Brussels: European Commission Directorate General III Industry.

European Commission (1999) Council Regulation (EC) 1493/ 1999 of 17 May 1999 on the common organisation of the market in wine. Official Journal of the European Communities L179, 1-84.

European Commission (2000a) Directive 2000/13/EC of the European Parliament and of the Council of 20 March 2000 on the approximation of the laws of the Member States relating to the labelling, presentation and advertising of foodstuffs. Official Journal of the European Communities L109, 29-42.

European Commission (2000b) Commission Regulation (EC) $1565 / 2000$ of 18 July 2000 laying down the measures 
necessary for the adoption of an evaluation programme in application of Regulation (EC) 2232/96 of the European Parliament and of the Council. Official Journal of the European Communities L180, 8-16.

European Commission (2000c) First Report on Harmonisation of Risk Assessment Procedures. Scientific Steering Committee Advising the European Commission in the Area of Human and Environmental Health. Brussels: European Commission.

European Commission (2002) Commission Decision 2002/113/ EC of 23 January 2002 amending Commission Decision 1999/ 217/EC as regards the register of flavouring substances used in or on foodstuffs. Official Journal of the European Communities L49, 1-160.

European Commission (2003) Directive 2003/89/EC of the European Parliament and of the Council of 10 November 2003 amending Directive 2000/13/EC as regards indication of the ingredients present in foodstuffs. Official Journal of the European Communities L308, 15-18.

European Commission (2005) Directive 2005/26/EC of the European Parliament and of the Council of 21 March 2005 establishing a list of food ingredients or substances provisionally excluded from Annex IIIa of Directive 2000/13/ EC of the European Parliament and of the Council. Official Journal of the European Communities L75, 33-34.

European Food Safety Authority (2004) Opinion of the Scientific Panel on Dietetic Products, Nutrition and Allergies on a request from the Commission related to a notification from AAC on wheat-based glucose syrups including dextrose pursuant to Article 6 paragraph 11 of Directive 2000/13/ECScientific Panel on Dietetic Products, Nutrition and Allergies (EFSA-Q-2004-091). http://www.efsa.eu.int/science/nda/nda opinions/681_en.html

EUROSTAT (2005) http://europa.eu.int/comm/eurostat

Food and Agriculture Organization (2003) FAO food balance sheets, 1999-2001. http://www.fao.org/documents/show_cdr. asp?url file=/DOCREP/006/Y5065M/Y5065M00.HTM

Food and Agriculture Organization/World Health Organization (2001) Pesticide Residues in Food-2000. Report of the Joint Meeting of the FAO Panel of Experts on Pesticide Residues in Food and the Environment and the WHO Core Assessment Group. FAO Plant Production and Protection Paper. Report no. 163. Rome: FAO.

Gilsenan MB \& Gibney MJ (2004) Assessment of the influence of energy under-reporting on intake estimates of four food additives. Food Additives and Contaminants 21, 195-203.

Hansen SC (1979) Conditions for use of food additives based on a Budget for an Acceptable Daily Intake. Journal of Food Protection 42, 429-434.

Huggett AC \& Hischenhuber C (1998) Food manufacturing initiatives to protect the allergic consumer. Allergy 53, Suppl. 46, 89-92.

Kroes R, Muller D, Lambe J, Lowik MR, van Klaveren J, Kleiner J, Massey R, Mayer S, Urieta I, Verger P \& Visconti A (2002) Assessment of intake from the diet. Food and Chemical Toxicology 40, 327-385.

Lawrie CA \& Rees NMA (1996) The approach adopted in the UK for the estimation of the intake of food additives. Food Additives and Contaminants 13, 411-416.

Leclercq C, Arcella D \& Turrini A (2000a) Estimates of the Theoretical Maximum Daily Intake of erythorbic acid, gallates, BHA and BHT in Italy. A stepwise approach. Food and Chemical Toxicology 38, 1075-1084.
Leclercq C, Molinaro MG, Piccinelli R, Baldini M, Arcella D \& Stacchini P (2000b) Dietary intake exposure to sulphites in Italy-Analytical determination of meals containing potential sulphite vectors. Food Additives and Contaminants 17, 979-989.

Legault L, Brandt MB, McCabe N, Adler C, Brown AM \& Brecher S (2004) 2000-2001 food label and package survey: an update on prevalence of nutrition labeling and claims on processed, packaged foods. Journal of the American Dietetic Association 104, 952-958.

Madsen C (2001) Where are we in risk assessment of food allergens? The regulatory view. Allergy 56, Suppl. 67, 91-93.

Mareschi JP, François-Collange M \& Suschetet M (1992) Estimation of sulphite in food in France. Food Additives and Contaminants 9, 541-549.

Munro IC, Kennepohl E \& Kroes R (1999) A procedure for the safety evaluation of flavouring substances. Joint FAO/WHO Expert Committee on Food Additives. Food and Chemical Toxicology 37, 207-232.

National Institute for Food and Nutrition Research (2003) Food labels database. Rome: INRAN.

NEVO Foundation (2004) Dutch Nutrient Database. Den Haag, The Netherlands: Voedingscentrum.

Ortolani C, Ispano M, Scibilia J \& Pastorello EA (2001) Introducing chemists to food allergy. Allergy 56, Suppl. 67, 5-8.

Ough CS (1986) Determination of sulphur dioxide in grapes and wines. Journal of the Association Official Analytical Chemistry 69, 5-7.

Petersen BJ (2000) Probabilistic modelling: theory and practice. Food Additives and Contaminants 17, 591-599.

Poms RE, Klein CL \& Anklam E (2004) Methods for allergen analysis in food: a review. Food Additives and Contaminants 21, 1-31.

Saba A, Turrini A \& Cialfa E (1992). Estimate of intakes: methodology and results of some studies carried out in Italy. Food Additives and Contaminants 9, 527-534.

Sampson HA (1999) Food allergy. Part 1: immunopathogenesis and clinical disorders. Journal of Allergy and Clinical Immunology 103, 717-728.

Sicherer SH, Munoz-Furlong A, Murphy R, Wood RA \& Sampson HA (2003) Symposium: Pediatric food allergy. Pediatrics 111, 1591-1594.

Taylor SL, Hefle SL, Bindslev-Jensen C, Bock SA, Burks AW Jr, Christie L et al. (2002) Factors affecting the determination of threshold doses for allergenic foods: how much is too much? Journal of Allergy and Clinical Immunology 109, 24-30.

TNO Nutrition and Food Research (2004) The Databank ALBA. www.allergenendatabank.nl

Trichopoulou A (2001) The DAFNE databank as a simple tool for nutrition policy. DAta Food NEtworking. Public Health Nutrition 4, 1187-1198.

van Klaveren JD (1999) Quality Programme for Agricultural Products. Results Residue Monitoring in The Netherlands. Wageningen, The Netherlands: RIKILT.

World Health Organization (1997) Food Consumption and Exposure Assessment of Chemicals. Food Consumption and Exposure Assessment of Chemicals. WHO/FSF/FOS/97 5. Geneva: FAO/WHO

Wuthrich B (2000) Lethal or life-threatening allergic reactions to food. Journal of Investigational Allergology and Clinical Immunology 10, 59-65. 